\title{
A Novel Method for Radio Propagation Simulation Based on Automatic 3D Environment Reconstruction
}

\author{
Danping HE, Guixuan LIANG, Jorge PORTILLA, Teresa RIESGO \\ Centro de Electronica Industrial, Universidad Politecnica de Madrid \\ Jose Gutierrez Abascal, 2, 28006 Madrid, Spain \\ danping.he@alumnos.upm.es
}

\begin{abstract}
In this paper, a novel method to simulate radio propagation is presented. The method consists of two steps: automatic $3 D$ scenario reconstruction and propagation modeling. For $3 D$ reconstruction, a machine learning algorithm is adopted and improved to automatically recognize objects in pictures taken from target regions, and $3 D$ models are generated based on the recognized objects. The propagation model employs a ray tracing algorithm to compute signal strength for each point on the constructed $3 D$ map. Our proposition reduces, or even eliminates, infrastructure cost and human efforts during the construction of realistic $3 D$ scenes used in radio propagation modeling. In addition, the results obtained from our propagation model proves to be both accurate and efficient.
\end{abstract}

\section{Keywords}

Image understanding, radio propagation, wireless communication, 3D reconstruction.

\section{Introduction}

Radio propagation modeling has been researched for decades in an effort to estimate signal strengths more accurately in wave propagation environments. Most of the adequately designed propagation models lack efficiency when a priory knowledge of the physical environment is not presented by a database. The accuracy of such propagation models has strong dependencies on the accuracy of the information captured from the database. The traditional reconstruction process, in which the environment is extracted meter by meter, is usually tedious and prolonged. Alternatively, people may purchase expensive digital maps from professional companies in order to save time and attempts on nonpropagation related issue. As a result, either time or money is needed in traditional methods. In order to make the whole simulation procedure time efficient and accurate with lower cost, a novel approach is proposed in this paper.

A simplified overview of the methodology is indicated in Fig. 1. It consists of two steps: 3D scenario reconstruction and radio propagation simulation. At the beginning, 3D environment database is automatically constructed from the pictures taken by hand-held camera or by satellite. The unsupervised image understanding algorithm is developed to recognize different objects in the images. The recognized objects are segmented and then vectorized to build the 3D environment database, in which the locations and materials of different objects are stored. This approach allows reconstructing large scale 3D maps at low computational complexity, high accuracy and low cost. In the second step, an improved ray-tracing propagation model is run on the generated 3D database to simulate the path loss for each point within the target region. The method not only considers the attenuation parameters of different materials but also considers the orders of obstacles along the signal path. The results from the proposed model indicate both efficiency and accuracy by comparing it to other advanced methods in the Munich scenario [1].

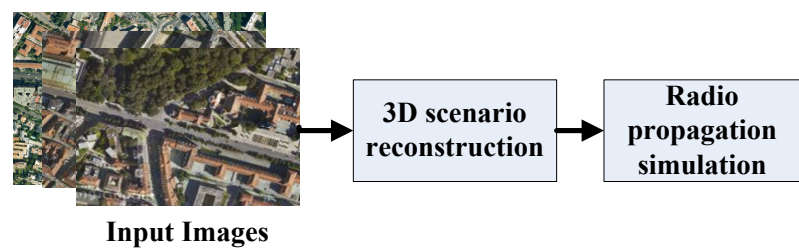

Fig. 1. Work flow of the proposed method.

The rest of this paper is organised as follows: The $3 \mathrm{D}$ environment reconstruction method is introduced in Section 2, and preliminary tests are evaluated on some image sets. In Section 3, we describe the ray-tracing based radio propagation model, from which the evaluation procedure is implemented on the Munich scenario. The results of simulation are compared with other related methods in Section 4. Finally, we state the conclusions and future work in Section 5 .

\section{3D Reconstruction Method}

A large part of physical signal degradation is caused by obstacles interfering with the signal path. In urban scenarios, obstacles have architectural bearing, whereas in indoor environments, walls, doors, desks or even humans are regarded as obstacles. In this paper, we focus on constructing large 


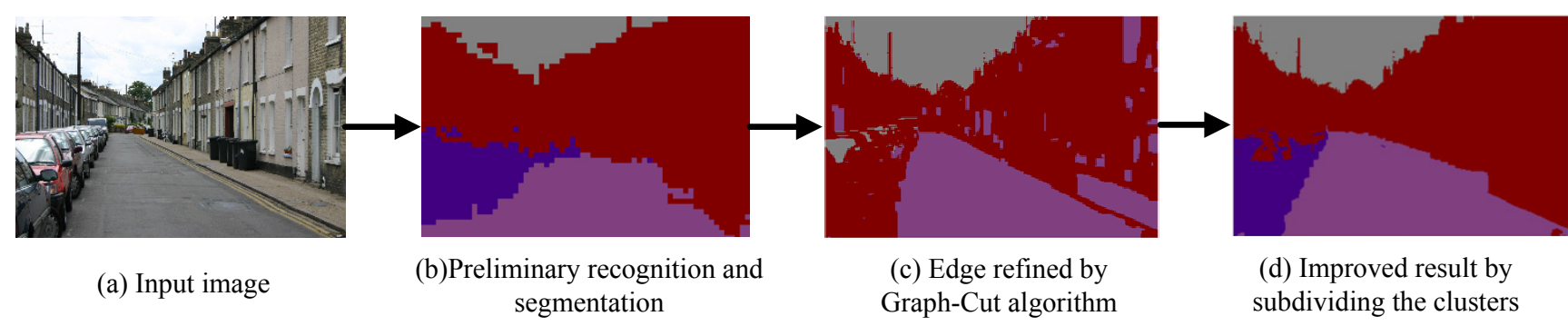

Fig. 2. Multi-object recognition procedure.

outdoor scenarios. As previously mentioned, our obstacles will not be manually labeled and measured as demonstrated in traditional method. Instead, they are reconstructed automatically in order to reduce time and efforts on the database reconstruction and constraints.

In [2] and [3], the identification of objects is made by a shape feature descriptor. The method in [4] uses color and texture features. Furthermore, [5] and [6] recognize different obstacles based on machine learning incorporating texture, layout, and context information. Our method employs and improves the machine learning procedure developed in [6]. Fig. 2 indicates the procedure of object recognition and segmentation. Given pictures taken from the target areas (from hand-held camera or satellite images such as those from Google Earth, see Fig. 2(a)), the preliminary results (see Fig. 2(b)) are obtained by adapting the Joint Boost algorithm [6] (JBA) which iteratively selects discriminative texture-layout filters $v_{[r, t]}(i)$ to compute weak learners, and combines them into a strong classifier of the form $H(c, i)=\sum_{m=1}^{M} h_{i}^{m}(c)$. Each weak learner $h_{i}(c)$ is a decision stump based on the feature response.

$$
h_{i}(c)= \begin{cases}a\left[v_{[r, t]}(i)>\theta\right]+b & \text { if } c<C, \\ k^{c} & \text { otherwise. }\end{cases}
$$

For those classes that share the feature $c \in C$, the weak learner gives $h_{i}(c) \in\{a+b, b\}$ depending on the comparison of the feature response to a threshold $\theta$. For classes not sharing the feature, the constant $k^{c}$ makes sure that unequal numbers of training examples of each class do not adversely affect the learning procedure. We use sub-sampling and random feature selection techniques for the iterative boosting [6]. The estimated confidence value can be reinterpreted as a probability distribution using soft max transformation [7] to give the texture layout potentials:

$$
P(c \mid x, i) \propto \exp H(c, i) .
$$

In Fig. 2(b), although most of the pixels are classified correctly, the edges between adjacent objects are not accurate enough. The authors in [6] solve this problem by manually indicating the misclassified parts, and then another classifier is used to adjust those pixels into the right classes. As it's intended to finish this procedure automatically in this work, the performance is improved by using color cues, which are frequently used for edge detection. A graph cut algorithm [8], [9] is employed to smoothly cut the edges based

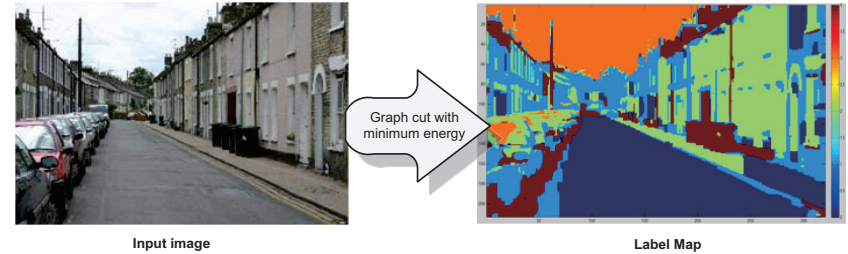

Fig. 3. Example of implementing K-means clustering and Graph Cut algorithm.

on cluster information. By using K-means clustering, the first stage identifies $k$ distinct clusters in the color space of the image. $k$ ranges from 5 to 8 depending on the complexity of the target area. Then each pixel is assigned to its cluster and the graph cut algorithm poses smoothness constraint on this labeling by employing expansion algorithm finds a labeling within a known factor of the global minimum. Hence a label map is generated as shown in Fig. 3. Dominant class is calculated by selecting maximum likelihood among all the classes for each cluster (see (3) and (4)),

$$
\begin{gathered}
P\left(c_{i} \mid k\right)=\frac{1}{\operatorname{area}(k)} \sum_{j \in k}\left[C(j)==c_{i}\right], \\
\operatorname{Class}_{G C}(k)=\operatorname{Max}\left(P\left(c_{i} \mid k\right)\right)
\end{gathered}
$$

where $P\left(c_{i} \mid k\right)$ is the likelihood of class $c_{i}$ given cluster $k$. $C(j)$ is the class label of pixel $j$ decided by TBA. $\operatorname{Class}_{G C}(k)$ is the class decision for cluster $k$. Therefore, the inaccurate edge issue is solved as shown in Fig. 2(c). However, several objects are misclassified because of the color similarity among different classes, for instance, the color of sky and that of the windshields of cars are similar, some parts of buildings and the road have similar colors. To tackle this problem, we further subdivide each cluster into variety numbers of sub-clusters based on the connectivity property (see Fig. 4). The selection of dominant class is repeated for each sub-cluster by using (3) and (4). At the end, objects are segmented and the final decision is obtained (see Fig. 2(d)).

To increase accuracy and reduce computational cost, researchers may choose a subset of labeled images that is the closest to the given testing sequence to train the classifier. In this work, the classifier will not be trained from the MSCR 21-class full labeled data which is too huge and consist of many redundant classes (e.g. animals, road signs, furnitures) that are trivial for outdoor environment. We performed experiments on a subset of the database, and only focus on 6 out of the 21 classes: building, grass, tree, sky, car, road, 


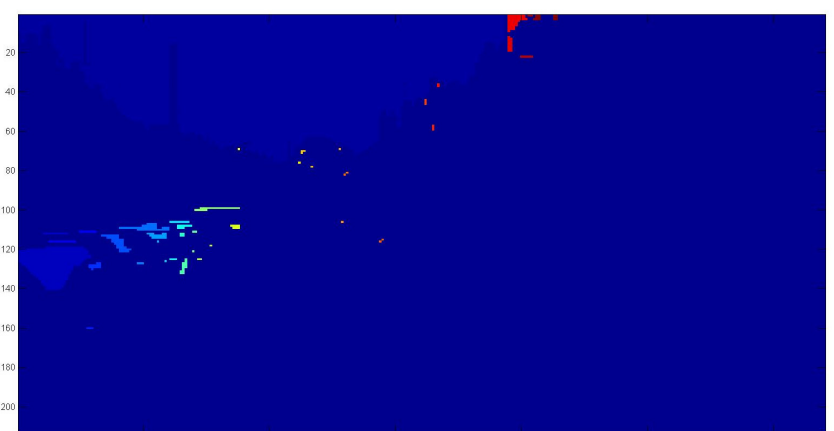

Fig. 4. Example of sub-clustering based on connectivity property.

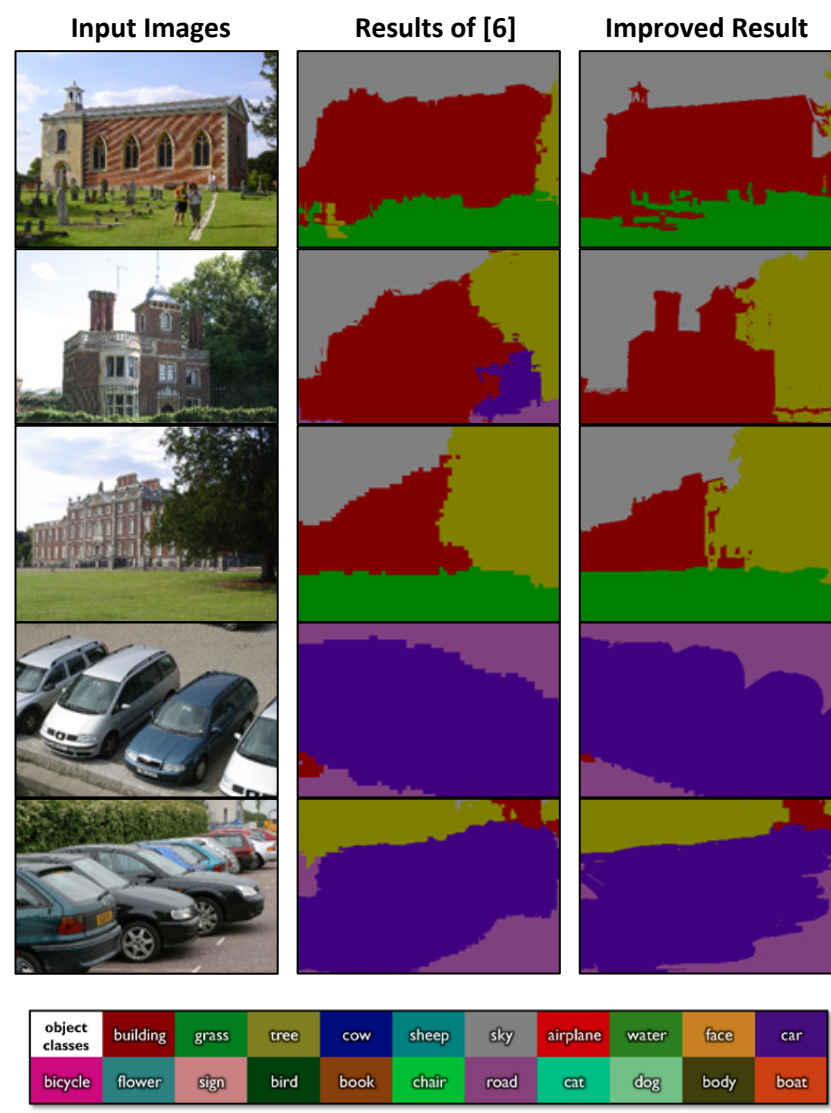

Fig. 5. Comparison of classification results.

\begin{tabular}{|c|c|c|c|c|c|c|}
\hline True class & 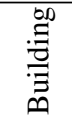 & 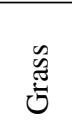 & $\stackrel{Ð}{\Xi}$ & $\frac{\vec{n}}{n}$ & تี & $\begin{array}{l}\overrightarrow{\widetilde{Z}} \\
\stackrel{0}{0}\end{array}$ \\
\hline Building & 75.7 & 0.1 & 8.1 & 4.9 & 9.5 & 1.7 \\
\hline Grass & 2.4 & 44.3 & 35.0 & 0 & 1.1 & 17.2 \\
\hline Tree & 16.8 & 0.6 & 71.6 & 8.9 & 1.8 & 0.3 \\
\hline Sky & 10.0 & 0 & 5.7 & 84.3 & 0 & 0 \\
\hline Car & 12.8 & 0 & 6.0 & 0.4 & 76.0 & 4.9 \\
\hline Road & 7.1 & 0 & 1.5 & 0 & 2.6 & 88.8 \\
\hline
\end{tabular}

Tab. 1. Confusion Matrix. The number of clusters in texton booster is 400 , the average accuracy is $76.1 \%$. which might be the main objects that affect outdoor radio propagations. The experiment results are given in Tab. 1 in a format of confusion matrix. With 1000 rounds of boosting $45 \%$ of training images in the database, the number of texton clusters is set to be 400 to achieve the best average accuracy of $76.1 \%$ whereas the JBA gives an overall accuracy of $69.2 \%$. The authors of [6] then manually moved the misclassified parts to the right classes and the improved result has an accuracy of $72.2 \%$. A small set of results is visually shown in Fig. 5. It is noticed that the developed method outperforms the JBA algorithm and the edges of adjacent objects are much more accurate and clearer. Moreover, the proposed method has the capability to adjust the misclassified pixels to the correct classes. For instance, in the second image, some pixels belonging to the building are recognized as car by JBA. The proposed method is able to change the decision and move the pixels to the correct classes, such as some are moved to the building and some are moved to trees. Hence, the proposed multi-object recognition method is fully automatic and more accurate, and it can be used to decide the location of objects and automatically assign the attenuate coefficient for each object, which will be useful during the simulation of radio propagation.

In order to utilize the aforementioned algorithm to construct the real propagation environment, we build a new training image set from randomly cut images from Google map. Each image has a size of $800 \times 550$ pixels and they are manually labeled with color codes and form the ground truth, as illustrated in Fig. 6, where only buildings, trees and roads are considered this time to ignore trivial obstacles in outdoor environment. Afterwards, the image set is trained by the proposed algorithm. It took around half an hour to finish 1000 rounds of boosting process for 7 images. A toy example of the reconstructed 3D scene is shown in Fig. 7, and the accuracy is $85 \%$.

\section{Radio Propagation Simulation}

\subsection{Computational Geometry Part}

Many methods are developed to simulate radio propagation, where the objective is to reduce computation time while maintaining good accuracy. Generally speaking, the ray-tracing algorithm have very high accuracy [10], [11], [12], while the computation load is also very high as the method tests every intersection along the ray path. When the scenario area becomes large, traditional methods might take longer than anticipated. Several algorithms are developed to overcome the aforementioned drawback by slightly reducing accuracy. Beam tracing algorithms [13] extends the raytracing algorithm to reduce intersection tests, as well as to overcome sampling problems. The dominant path tracing algorithm in [14] is developed to avoid redundant calculation, since the authors believe that $98 \%$ of the received power is contributed by only a few radio rays. The ray tube tree 

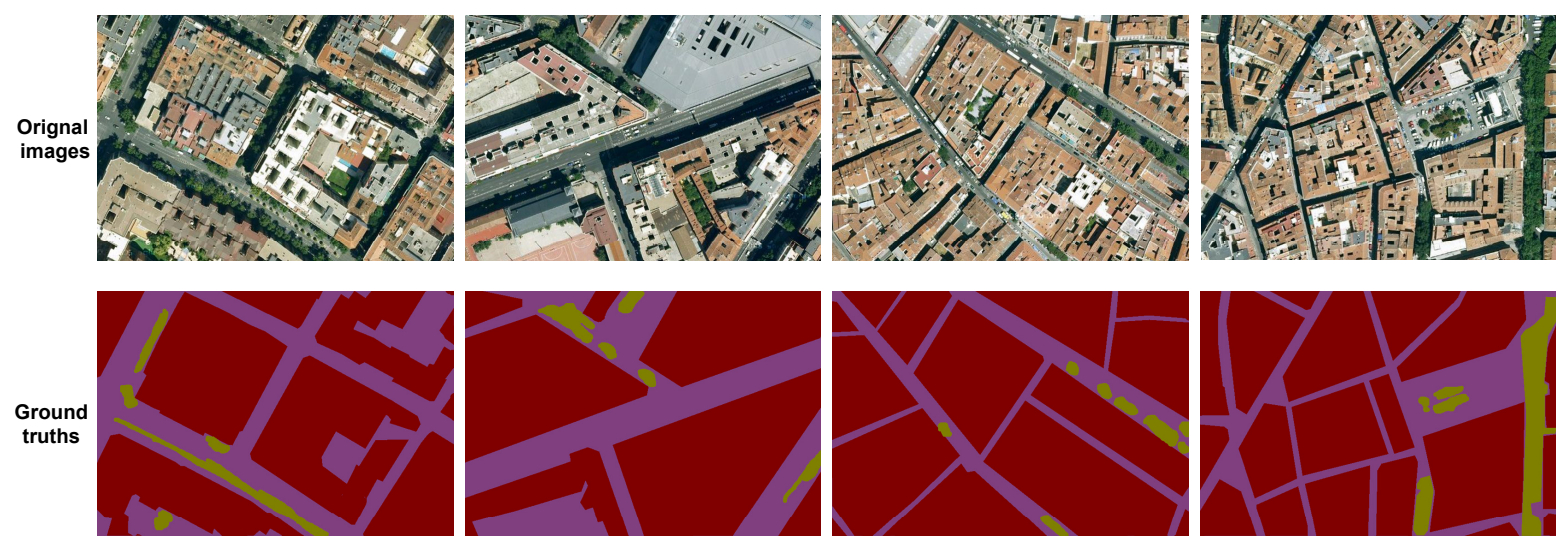

Fig. 6. Training image set and the ground truths.

method described in [15] increases the preprocessing speed in constructing trees for ray-tracing.

Our method takes advantage of automatic map reconstruction described in Section 2 by utilizing the ray-tracing algorithm. All the ray paths are calculated based on image concept which is more efficient than traditional geometry method. Basically, there are three types of rays: direct ray, reflected ray and diffracted ray. Other rays such as overrooftop ray and ground reflected ray are not discussed here. The roofs of buildings are assumed flat and thickness of wall is uniform. Each object is labeled with unique index to ease ray tracing procedure.

The algorithm searches for a direct path at each location. All the obstacles which intersects the transmitted ray in the Tx-to-Rx path, will provoke signal power attenuation. For a location $q$, the direct path loss information is stored as $L_{q}=\left[a_{1}, a_{2}, a_{3}, \cdots, a_{n}\right], a_{1} \sim a_{n}$ are the attenuation parameters for the corresponding obstacles sorted along the path from Tx to $q$.

After all the direct paths have been calculated, each location in the target region has $L_{q}, q \in$ targetregion, $L_{q}$ not only provides path loss information, but its length also indicates the number of obstacles that exist between Tx and $q$. Based on the length of $L_{q}$ at each location $q$, visible segments from Tx are swept by selecting pixels belonging to objects with length $\left(L_{p}\right)=1$. Reflections happen on the visible edges of those visible segments.

Traditionally, maps offer structural information, including vertex locations for planes and polygons, and material property of each obstacle. However, in this work, the map is automatically constructed from images, although objects are correctly labeled, feature points to represent polygons are not calculated. One of the benefits of this method is that only the visible segments are needed to be vectorized, which reduces redundant calculation for the whole map. The Canny edge detector is employed to detect visible lines along each visible segment, and the Harris corner detector searches the feature points for each visible line, by combining the feature points and edge information together we have pairs of vertexes representing visible vectorized lines. Reflected

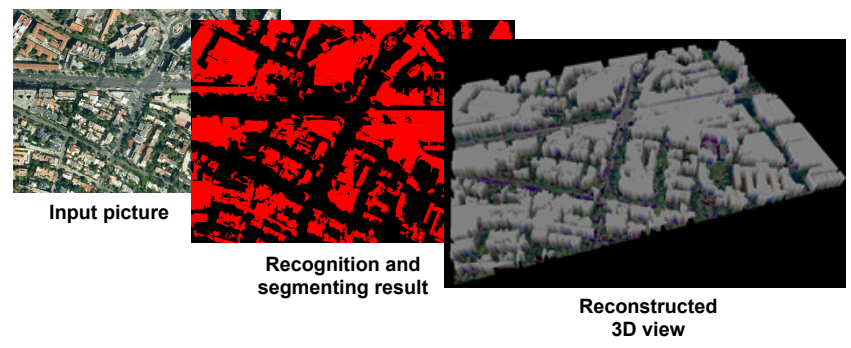

Fig. 7. 3D reconstruction based on object recognition and segmentation.

sources are calculated by mirroring the TX along this visible line. The details are introduced below:

1. Visible segments are highlighted in the image, others are considered as background.

2. Canny edge detector is tested on each visible segment, the visible lines are obtained in the image.

3. Harris corner detector is used to find feature points along detected edges, and $V_{i}$ is used to represent position of the feature point $i$. Edge is expressed by a pair of vertexes of the form $E\left(V_{i}, V_{j}\right)$.

4. Reflected image sources are calculated by mirroring Tx along the edges, and stored as Reflections = $\left(x_{1}, y_{1}\right),\left(x_{2}, y_{2}\right),\left(x_{3}, y_{3}\right), \cdots,\left(x_{n}, y_{n}\right)$ for Tx, $n$ is limited by the maximum reflection number.

5. Each virtual point serves as new transmitter, and the whole procedure is repeated until reaches the maximum iteration number.

All the reflected points are seen as virtual sources with same transmission power as real source, while with constraints in transmission angle. As shown in Fig. 8, the reflection region is constrained by combining maximum transmission range and angle $\angle V_{1} O^{\prime} V_{2}$.

According to the Geometrical Theory of Diffraction [16], diffracted rays are produced by incident rays which hit edges, corners or vertices of boundary surfaces. All the vertices and corners are obtained as a form of $V_{i}$ while searching 
for the reflected rays. Therefore, those vertices are automatically introduced as diffracted virtual sources. The diffracted region is also determined.

\subsection{Radio Propagation Model}

A radio propagation model is developed in this paper to estimate the power loss between the Tx and Rx points. The developed model can be used to calculate and produce a path loss profile of the transmitted signal, reflected and diffracted signal respectively by

$$
\begin{gathered}
L_{p}=10 \log _{10} d+L_{\text {obstacle }}, \\
L_{\text {obstacle }}=\sum_{i=1}^{N} l(i) \alpha(i)^{i-1}
\end{gathered}
$$

where $n$ is path loss coefficient with range between 2 and 5 . The value of $n$ is decided by the environment, i.e. in free space $n=2$, in others such as urban or rural environments $2<n<5$. For a direct path $d$ is the distance between TX and RX, for a reflected path and diffracted path, $d$ is the distance between the virtual source and RX. $L_{\text {obstacle }}$ is the power loss due to obstacles encountered on the signal path. This is calculated by accumulating the power reduction of each obstacle along the path. $l(i)$ is attenuation exponent of the $i^{\text {th }}$ obstacle, and $\alpha(i)$ ranges from 0 to 1 , which is the penetration rate of the material of $i^{t h}$ obstacle. $\alpha(i)^{i-1}$ decreases when $i$ increases, which means that the first object, with which the signal intersects, produces the most significant power loss. Fig. 9 gives an example of calculating (6) in the direct path. The signal first penetrates through a glassmade object, then through a concrete obstacle. By assuming that $l(1)=2 \mathrm{~dB}, l(2)=23 \mathrm{~dB}$, we can set $\alpha=0.9$ as a constant value, we then have $L_{\text {obstacle }}=2+23 \times 0.9=22.7 \mathrm{~dB}$. Equation (5) indicates that not only distance, but also the material property of obstacles and their orders can affect the received signal strength. At the end, the power loss at the receiver is the accumulated value among the directed, reflected and diffracted paths.

\section{Experimental Results}

The proposed method was verified by comparing the results to that of Munich scenario [1], which was comprehensively studied by the European COST 231 working group. Fig. 10 illustrates the top view of the target region in Munich city. The size of the region is $2400 \mathrm{~m} \times 3400 \mathrm{~m}$ and there are 3 different routes measured by COST 231 group (e.g. the red color is the first route named as METRO200, the black one is the second route METRO 201, and the green one is the third rout METRO 202).

A new training database is created through similar methods described in Section 2. The training images are fetched randomly from Google Maps within the region of Munich city. Buildings, roads and trees are labeled in order to guarantee the learning of reliable and transferable knowledge. Fig. 11 shows the evaluation procedure: At the begin-

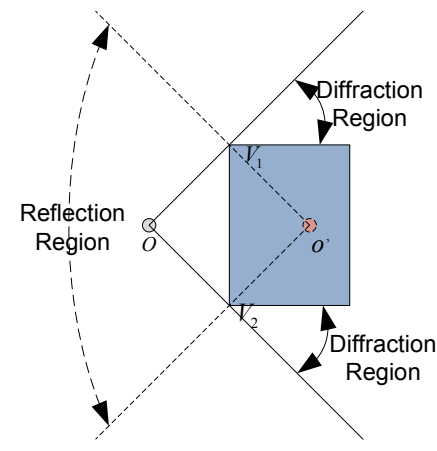

Fig. 8. Reflection region and diffraction region.
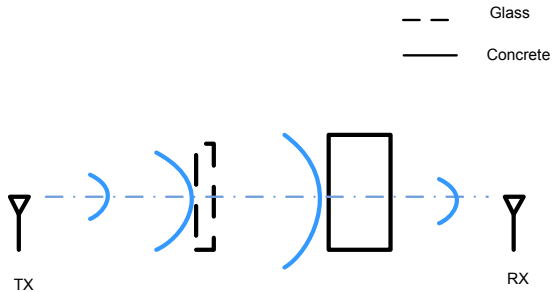

Fig. 9. Example of propagation.

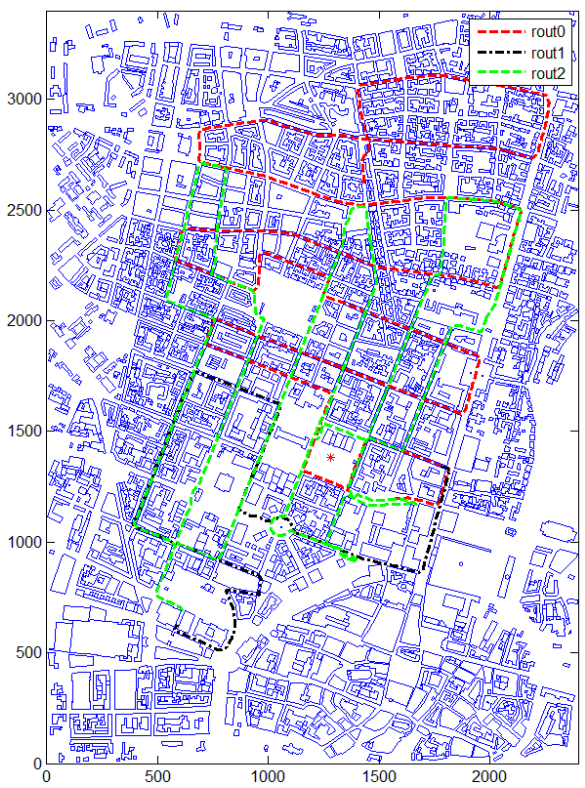

Fig. 10. Building geometry of Munich city and 3 different routes that measured by COST 231 group.

ning, the same area in which the COST 231 group did measurements is captured on Google map. The images are downloaded and divided into small sub-images, each of which is of the same size as the training images.

Afterwards, they are passed through the image classifier to recognize and segment the three objects. The recognized objects are then vectorized and the $3 \mathrm{D}$ database is automatically generated. Then the propagation simulator is run to simulate the signal transmission in the target area. Finally, the signal coverage map is visually presented to the user.

The proposed method is programmed by combining $\mathrm{C \#}$, Matlab and $\mathrm{C}++$ code. It was run on a PC equipped with 


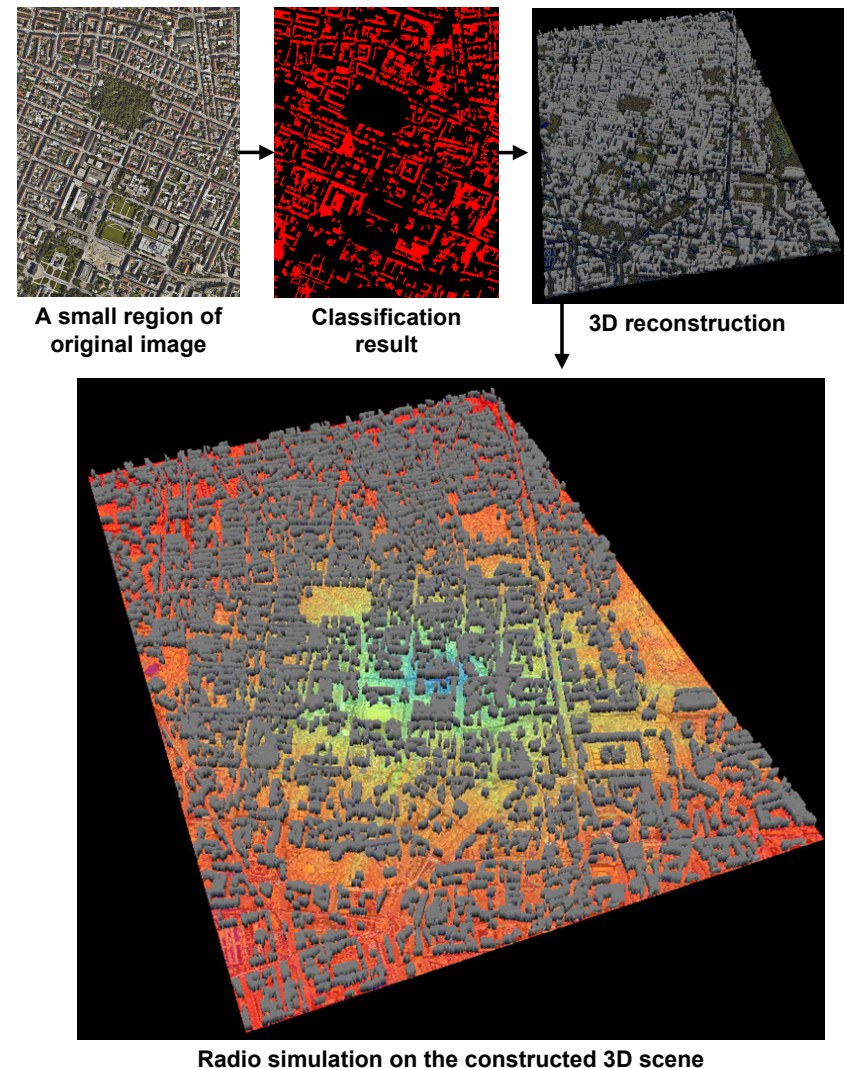

Fig. 11. Demonstration of the whole procedure in Munich city.

Intel i5 CPU, 2.8 GHz. It took less than 1 hour to construct the $3 \mathrm{D}$ database and approximately 15 minutes to finish radio estimation with a resolution of $1 \mathrm{~m}$. Hence it took around $0.3 \mathrm{~s}$ to process 2356 points for all the three routes.

The simulation results are compared with the practical measurements. As shown in Fig. 12, the results of the 3 different routes correlate well with the real measurements. The mean error (ME) and the standard deviation (STD) of the results are the two metrics used to evaluate the accuracy of the simulation method. The ME of METRO 200 is $-0.3 \mathrm{~dB}$ and the STD is $5.7 \mathrm{~dB}$. The ME is $-0.4 \mathrm{~dB}$ and STD is $5.5 \mathrm{~dB}$ in METRO 201. The ME and STD of METRO 202 are $2.2 \mathrm{~dB}$ and $6.7 \mathrm{~dB}$ respectively. The performances on the first two routes are similar and are better than the third route which has more measured points and some are located in the streets with much more buildings.

Tab. 2 compares the proposed method with other stateof-the-art technologies. The STD and ME of the proposed method are the smallest on METRO 200 and METRO 201, while they are slightly greater than CNET in METRO 202. The experimental results indicate that this novel approach has good efficiency and accuracy. A quantitative explanation for this improvement can be directed towards three reasons: the construction accuracy of the environment database is $82 \%$, the miss-classified pixels are $18 \%$. The resolution of simulation is $1 \mathrm{~m}$ which is higher than in other methods. Furthermore, the propagation model is adequate in describing reduced dimensionality environments, and most of the

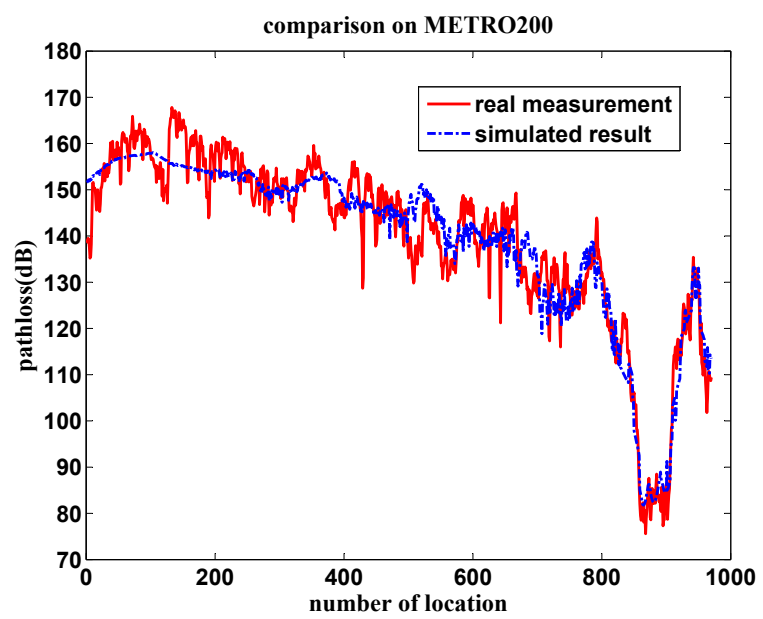

(a)

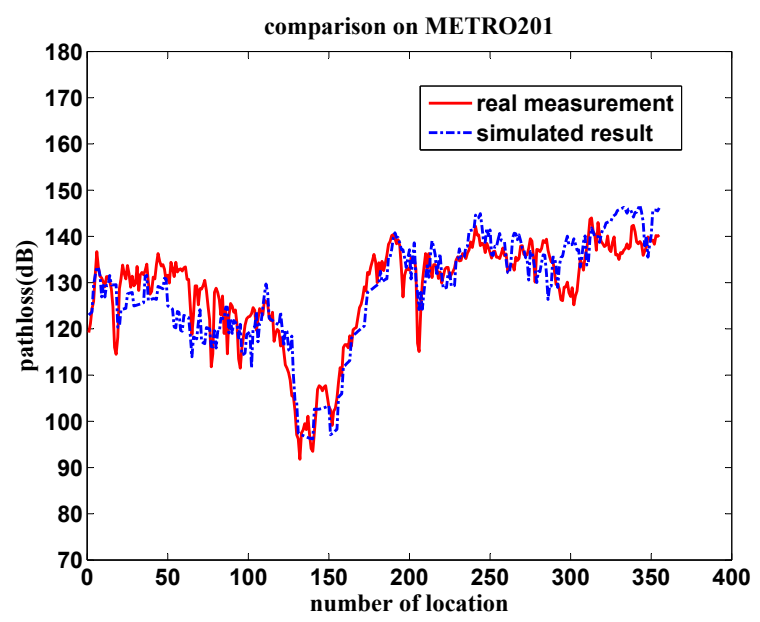

(b)

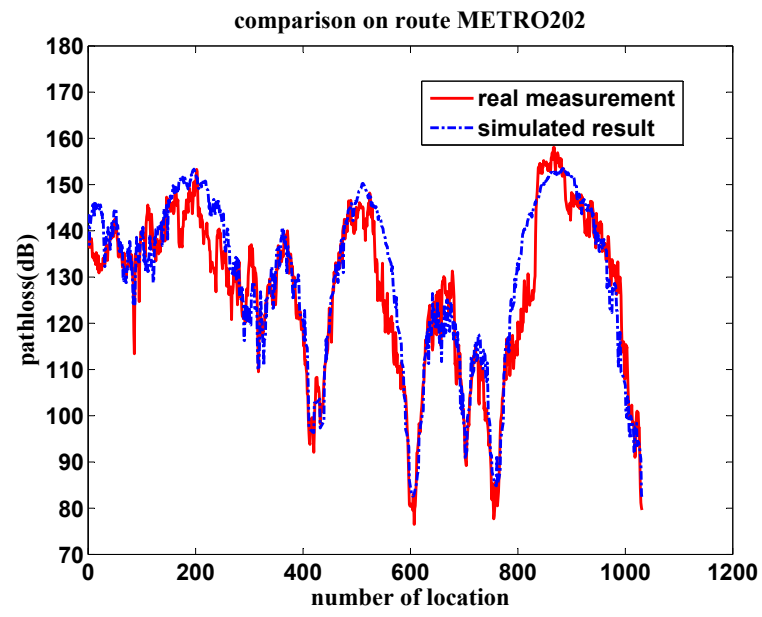

(c)

Fig. 12. Comparisons with measured path loss along 3 different routes in Munich city.

calculations used in the radio propagation model are based on efficient image manipulation rather than slow geometrical computation. 


\begin{tabular}{|c|r|r|r|r|r|r|}
\hline \multirow{2}{*}{ Method } & \multicolumn{2}{|c|}{ METRO200 } & \multicolumn{2}{|c|}{ METRO201 } & \multicolumn{2}{c|}{ METRO202 } \\
\cline { 2 - 7 } & STD & ME & STD & ME & STD & ME \\
\hline This paper & 5.7 & -0.3 & 5.5 & -0.4 & 6.7 & 2.2 \\
\hline Ericsson & 6.7 & 0.3 & 7.1 & 2.3 & 7.5 & 1.4 \\
\hline CNET & 6.9 & -2.1 & 9.5 & -3.6 & 5.6 & -0.2 \\
\hline COST-WI & 7.7 & 10.8 & 5.9 & 15.4 & 7.3 & 16.3 \\
\hline RAY-TRI[17] & 7.1 & -2.6 & 6.2 & -0.7 & 8.3 & -1.4 \\
\hline
\end{tabular}

Tab. 2. Comparison with other methods.

\section{Conclusion and Future Works}

In conclusion, a novel propagation simulation method was developed, tested and presented in this paper. Conceptually, to the best of our knowledge, this is the first method which utilizes the advantages of image understanding algorithm in order to automatically reconstruct 3D outdoor scenarios for radio propagation simulation. The experimental results indicate that the algorithm accurately recognizes different objects from the images of the target regions, thereby, reducing human efforts on reconstructing a $3 \mathrm{D}$ geographic database. Furthermore, the results indicate excellent correlation between the simulation and actual measurements. The developed propagation simulation method contributes on efficient calculation by using image processing concepts, while maintaining accuracy on the estimated results. This simulator is not only suitable for urban scenes but also suitable for indoor environments, since the learning algorithm can easily be trained for indoor object features. Therefore, the future work will include the development of methods to improve the flexibility and applicability of this approach to indoor specific environments.

\section{Acknowledgements}

The authors would like to acknowledge the support of ARTEMIS JU and Spanish Ministry of Industry and commerce for WSN DPCM project under grant ART-0100002011-1. The authors would also like to thank Jaco du Toit for his assistance during the review process.

\section{References}

[1] DAMOSSO, E. Digital Mobile Radio Towards Future Generation Systems. Final report of COST 231. Brussels (Belgium): European Commission, 1999.

[2] HE, L., LIU, H. Shape context for image understanding. In Proceedings of the 5th WSEAS International Conference on Signal, Speech and Image Processing. Corfu (Greece), 2005, p. 276 - 281.

[3] BELONGIE, S., MALIK, J., PUZICHA, J. Shape matching and object recognition using shape contexts. IEEE Transactions on Pattern Analysis and Machine Intelligence, 2002, vol. 24, no. 24, p. 509 522.

[4] ARIVAZHAGAN, S., SHEBIAH, R., NIDHYANANDHAN, S., GANESAN, L. Fruit recognition using color and texture features.
Journal of Emerging Trends in Computing and Information Sciences, 2010, vol. 1, no. 2 , p. 90 - 94

[5] HE, X., ZEMEL, R.S., CARREIRA-PERPINAN, M. Multiscale conditional random fields for image labeling. In Proceedings of IEEE Conference on Computer Vision and Pattern Recognition. Washington, DC (USA), 2004, vol. 2, p. 695 - 702.

[6] SHOTTON, J., WINN, J., ROTHER, C., CRIMINISI, A. TextonBoost for image understanding: multi-class object recognition and segmentation by jointly modeling texture, layout, and context. International Journal of Computer Vision, 2009, vol. 81, no. 1, p. 2 - 23.

[7] FRIEDMAN, J., HASTIE, T., TIBSHIRANI, R. Additive logistic regression: a statistical view of boosting. The Annals of Statistics, 2000, vol. 38, no. 2, p. 337 - 407.

[8] BOYKOV, Y., VEKSLER, O., ZABIH, R. Fast approximate energy minimization via graph cuts. IEEE Transactions on Pattern Analysis and Machine Intelligence, 2001, vol. 20, no. 12, p. 1222 - 1239.

[9] BOYKOV, Y., KOLMOGOROV, V. An experimental comparison of min-cut/max-flow algorithms for energy minimization in vision. IEEE Transactions on Pattern Analysis and Machine Intelligence, 2004, vol. 26, no. 9, p. 1124 - 1137.

[10] SCHAUBACH, K. R., DAVIS, N. J., RAPPAPORT, T. S. A ray tracing method for predicting path loss and delay spread in microcellular environments. In Proceedings of IEEE Vehicular Technology Conference. Denver (CO, USA), 1992, p. 932 - 935.

[11] SCHMITZ, A., KOBBELT, L. Wave propagation using the photon path map. In Proceedings of International Workshop on Performance Evaluation of Wireless Ad Hoc, Sensor and Ubiquitous Networks. Terromolinos (Spain), 2006, p. 158 - 161.

[12] KIM, S., GUARINO, B., WILLIS III, T., ERCEG, V., FORTUNE, S., VALENZUELA, R., THOMAS, L., LING, J., MOORE, J.D. Radio propagation measurements and prediction using three-dimensional ray tracing in urban environments at $908 \mathrm{MHz}$ and $1.9 \mathrm{GHz}$. IEEE Transactions on Vehicular Technology, 1999, vol. 48, no. 3, p. 931 946.

[13] SCHMITZ, A., RICK, T., KAROLSKI, T., KOBBELT, L., KUHLEN, T. Beam tracing for multipath propagation in urban environments. In Proceedings of $3^{\text {rd }}$ European Conference on Antennas and Propagation. Berlin (Germany), 2009, p. 2631 - 2635.

[14] WAHL, R., WOLFLE, G. Combined urban and indoor network planning using the dominant path propagation model. In Proceedings of $1^{\text {st }}$ European Conference on Antennas and Propagation. Nice (France), 2006, p. 1 - 6

[15] SON, H., MYUNG, N. A deterministic ray tube method for microcellular wave propagation prediction model. IEEE Transactions on Antennas and Propagation, 1999, vol. 47, no. 8, p. 1344 - 1350.

[16] KELLER, J. B. Geometrical theory of diffraction. Journal of the Optical Society of America, 1962, vol. 52, no. 2, p. 116 - 130.

[17] YUN, Z., ZHANG, Z., ISKANDER, M. F. A ray-tracing method based on the triangular grid approach and application to propagation prediction in urban environments. IEEE Transactions on Antennas and Propagation, 2002, vol. 50, no. 5, p. 750 - 758.

\section{About Authors ...}

Danping HE was born in Guangxi, China, in 1985. She received her B.E. from Huazhong University of Science and Technology in 2008, and the M.Sc. degree from the Universite Catholique de Louvain (UCL), Belgium and Politecnico di Torino (PdT), Italy, in 2010. Right now she is a Ph.D. student in Universidad Politecnica de Madrid. Her current 
research interests include wireless communication, propagation analysis, wireless sensor networks and topology control.

Guixuan LIANG was born in Nei Mongol, China, in 1985. He received his B.E. from Huazhong University of Science and Technology in 2008. After that he received his M.Sc. from the Hong Kong University of Science and Technology in 2009. Right now he is a Ph.D. candidate in Universidad Politecnica de Madrid. His research interests include MBOFDM Ultra wideband (UWB) digital baseband implementation, hardware/software co-simulation, digital signal processing, wireless communication, low-power digital system design and so on.

Jorge PORTILLA was born in Madrid, in 1978. He received the M.Sc. degree on Physics from the Universidad Complutense de Madrid (UCM) in 2003 and the Ph.D. de- gree in Electronic Engineering from the Universidad Politecnica de Madrid (UPM), Spain, in 2010, where he is presently an Assistant Professor since 2006, and doing his research at the Industrial Electronics Centre (CEI). His research interests are focused mainly in wireless sensor networks, reconfigurable systems and embedded systems design.

Teresa RIESGO was born in Madrid in 1965. She received her M.Sc. and Ph.D. degrees in Electrical Engineering from UPM, in 1990 and 1996, respectively. Since 2003 she is Full Professor of Electronics at UPM. She has been the Director of the Center of Industrial Electronics (CEI-UPM) from 2007 to 2011. She has acted as General and Program Chair of different conferences in the field of Electronic system design and she has published many papers in journals and conferences in the field. Her research interests are focused in embedded system design, reconfigurable systems and wireless sensor networks. 